\title{
Transposon-induced gene activation as a mechanism generating cluster shape somatic variation in grapevine
}

\author{
Lucie Fernandez ${ }^{1,2}$, Laurent Torregrosa ${ }^{3}$, Vincent Segura ${ }^{4}$, Alain Bouquet ${ }^{3, \dagger}$ and José M. Martinez-Zapater ${ }^{1,2, *}$ \\ ${ }^{1}$ Departmento de Genética Molecular de Plantas, Centro Nacional de Biotecnología, CSIC, C/Darwin 3, 28049 Madrid, Spain, \\ ${ }^{2}$ Instituto de Ciencias de la Vid y del Vino (ICVV), (CSIC, Universidad de La Rioja, Gobierno de La Rioja), CCT, Campus de la \\ Universidad de La Rioja, C/Madre de Dios 51, 26006 Logroño, Spain, \\ 3 INRA-SupAgro, UMR DIA-PC, Campus SupAgro/INRA, 2 Place P. Viala, 34060 Montpellier Cedex, France, and \\ ${ }^{4}$ Department of Biology, Centre for Novel Agricultural Products, University of York, PO Box 373, York YO10 5YW, UK
}

Received 12 August 2009; revised 17 October 2009; accepted 2 November 2009; published online 4 January 2010.

*For correspondence (fax + 34 915854506; e-mail zapater@cnb.csic.es).

${ }^{\dagger}$ Our friend and colleague Alain Bouquet, who actively participated in this work and co-authored this manuscript, passed away unexpectedly during its final writing. He enjoyed a very productive scientific life, dedicated to the biology of grapevine and its genetic improvement, and he was an enthusiastic and wonderful person, professor and colleague. We dedicate this article to his memory.

\begin{abstract}
SUMMARY
We have characterized the genetic and molecular origin of the reiterated reproductive meristem (RRM) somatic variant phenotype of grapevine cultivar Carignan. Here, we show that the extreme cluster proliferation and delayed anthesis observed in this somatic variant is caused by a single dominant mutation. Transcriptional profiling of Carignan and RRM plants during early stages of inflorescence development demonstrated the overexpression of a few regulatory genes, including VvTFL1A, a close TFL1 Arabidopsis homolog, in RRM inflorescences. Genetic and molecular analyses correlated the insertion of a class-Il transposable element, Hatvine1-rrm, in the VvTFL1A promoter, with upregulation of the corresponding VvTFL1A allele in reproductive and vegetative organs of the shoot apex. These results suggest a role for this TFL1 grapevine homolog in the determination of inflorescence structure, with a critical effect on the size and branching pattern of grapevine fruit clusters. Our results demonstrate the existence of spontaneous cis-activation processes caused by class-II transposable elements in grapevine plants, and point to their possible role as a mechanism to generate somatic cell variation in perennial plants. This mechanism is expected to generate dominant phenotypes in chimeric sectors that can be readily exposed to natural selection.
\end{abstract}

Keywords: inflorescence development, inflorescence structure, somatic variation, active transposition, grapevine.

\section{INTRODUCTION}

Fruit and forest trees are perennial plant species with long generation times, which has influenced their domestication, mostly by the selection and vegetative propagation of elite genotypes (Zohary, 2004). Long generation times facilitate the accumulation of genetic variation, affecting somatic cells that, given the lack of germ line-characterizing plants, can be transmitted to the next sexual generation (Walbot and Evans, 2003). Only a small proportion of the spontaneous somatic variation is phenotypically expressed within the sporophytic generation, given that mutant somatic cells are frequently heterozygous for recessive mutations, and present as chimeric sectors within the plant. Therefore, only gain-of-function mutations or loss-of-function mutations causing haploinsuficiency will generate a phenotype under these conditions.

Somatic variation is important for genetic improvement in fruit trees, such as Citrus species (Moore, 2001) or grapes (This et al., 2006), where vegetative reproduction is used to propagate new interesting phenotypes, appearing as spontaneous sports. In spite of its relevance, little is known about the genetic and epigenetic mechanisms causing this variation. From the time of grapevine (Vitis vinifera) domestication, dated to approximately 8000 years ago (McGovern et al., 1986), the selection of elite genotypes and their vegetative propagation has been a common approach for cultivar improvement and management (Zohary and Hopf, 
2000). Throughout grapevine domestication multiple spontaneous somatic variants have been selected in different cultivars for physiological and berry traits, such as earliness, cluster and berry size, seedlessness, berry taste, berry colour, etc. (This et al., 2006). However, the molecular basis of this phenotypic variation is only known for two traits. On one hand, the cultivar Pinot Meunier, displaying tomentose vegetative organs when compared with its original cultivar, Pinot Noir, was shown to be a periclinal chimera carrying a gain-of-function mutation in $V v G A / 1$ in the L1 cell layer, which impairs gibberelic acid signaling (Boss and Thomas, 2002). On the other hand, red color berry variants, quite frequent in white berry cultivars, have been shown to appear as a result of recombination between long terminal repeats (LTRs) of the Gret1 retrotransposon, present in the homozygous state at the promoter of $M y b A 1$ in white cultivars (Kobayashi et al., 2004; Lijavetzky et al., 2006).

Grapevine somatic variants can also be useful tools for the study of gene function, because they result from the effect of single mutation events in a given genetic background. In this way, characterization of the fleshless somatic variant in cultivar Ugni Blanc has provided new tools to study ovary and fruit morphogenesis (Fernandez et al., 2006, 2007). In addition, other somatic variants altered in reproductive development have been described, showing the possibilities of this type of natural variation in the study of woody plant development (Chatelet et al., 2007). Among those variants, the reiterated reproductive meristems (RRM) somatic variant of cultivar Carignan exhibits an early phenotypic alteration of inflorescence development, and a delayed anthesis that affects fruit cluster development and ripening time (Chatelet et al., 2007). Fruit cluster size and compactness as well as anthesis time and berry ripening are all traits of interest in grape production, either for wine making or direct fruit consumption.

Here, we have investigated the genetic and molecular origin of the RRM phenotype to shed light on the process of grapevine inflorescence and fruit cluster development, and to understand the molecular mechanisms responsible for this spontaneous phenotypic variation. Using a combination of genetic, transcriptomic and molecular approaches, we show that reiteration of reproductive meristems results from a spontaneous cis-activation of $V V T F L 1 A$, the grapevine homolog of Arabidopsis TFL 1, suggesting a critical role of VVTFL 1A in the proliferation and branching of inflorescences and tendril meristems in grapevine. Furthermore, we show that this cis-activation is caused by a new insertion of a classII transposon in its promoter region, which seems to enhance its expression. Spontaneous 'activation tagging' mediated by endogenous transposons could represent a genetic mechanism to generate somatic variation that can be readily exposed to natural selection during the life of perennial species, contributing to their adaptation and evolution.

\section{RESULTS}

\section{Morphological characterization of the RRM somatic variant}

Reproductive development. The most conspicuous phenotype of the RRM somatic variant was the production of larger inflorescences, as well as a 1-month delay in the time of anthesis, with respect to the original Carignan plant (Figure 1a,b). This delay did not result from late flowering induction, as the analysis of shoot development showed that RRM plants developed the first inflorescence at an equivalent node position as Carignan, and produced a similar number of inflorescences per shoot (data not shown).

The RRM inflorescences also produced a higher number of flowers per floral group than Carignan plants. These flower groups developed asynchronously, showing a gradient, from flower meristem to pea-size berries, that is never observed in the reproductive development of grapevine plants (Figure 1c,d).
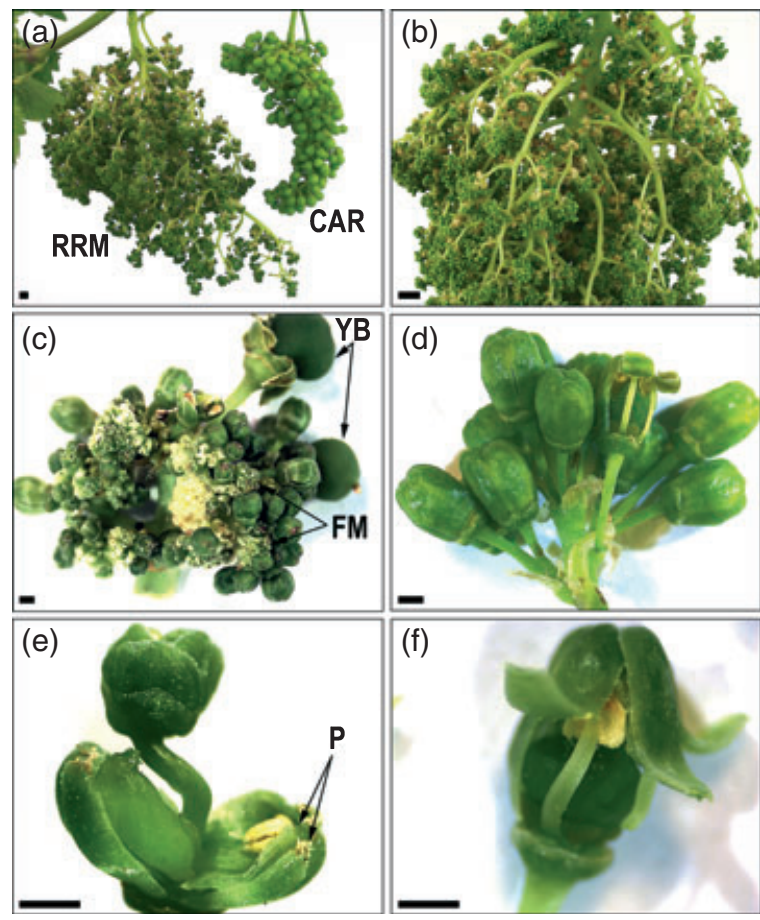

Figure 1. Macroscopic phenotype of the reiterated reproductive meristems (RRM) somatic variant inflorescences and flowers.

(a) Comparison of the RRM and Carignan (CAR) inflorescences at the same date.

(b) Close-up of the ramose structure of the RRM inflorescence.

(c) Inflorescence apex, showing the presence of young berries (YB), flowers at several stages of development and the reiterated production of flower meristems (FM).

(d) Inflorescence apex in CAR plants showing a typical dichasium group of flowers synchronically developed.

(e) Reiteration of petal whorls (P) and formation of one additional flower within the flower.

(f) Regular CAR flower at anthesis.

Scale bars: $1 \mathrm{~cm}$ in (a) and (b); $1 \mathrm{~mm}$ in (c-f) 
Figure 2. Microscopic analyses of the reiterated reproductive meristems (RRM) inflorescence and flower development.

Developmental stage in winter latent buds reached by RRM inflorescences (a) and Carignan (CAR) inflorescences (b).

Developmental stage in bursting buds of RRM inflorescences (c) and CAR inflorescences and flowers (d).

(e) RRM inflorescence and flower meristems a stage IS1.

(f) CAR flower development during stage IS1. $A$, anther; $B$, bract; $B B$, bud burst; $F M$, flower meristem; IB, inflorescence branch; IBM, inflorescence branch meristem; IM, inflorescence meristem; IS1, inflorescence stage $1 ; \mathrm{P}$, petal; $\mathrm{S}$, sepal; SAM, shoot apical meristem; WB, winter buds.

Scale bars: $100 \mu \mathrm{m}$ in $(a-c) ; 250 \mu \mathrm{m}$ in (d-f).
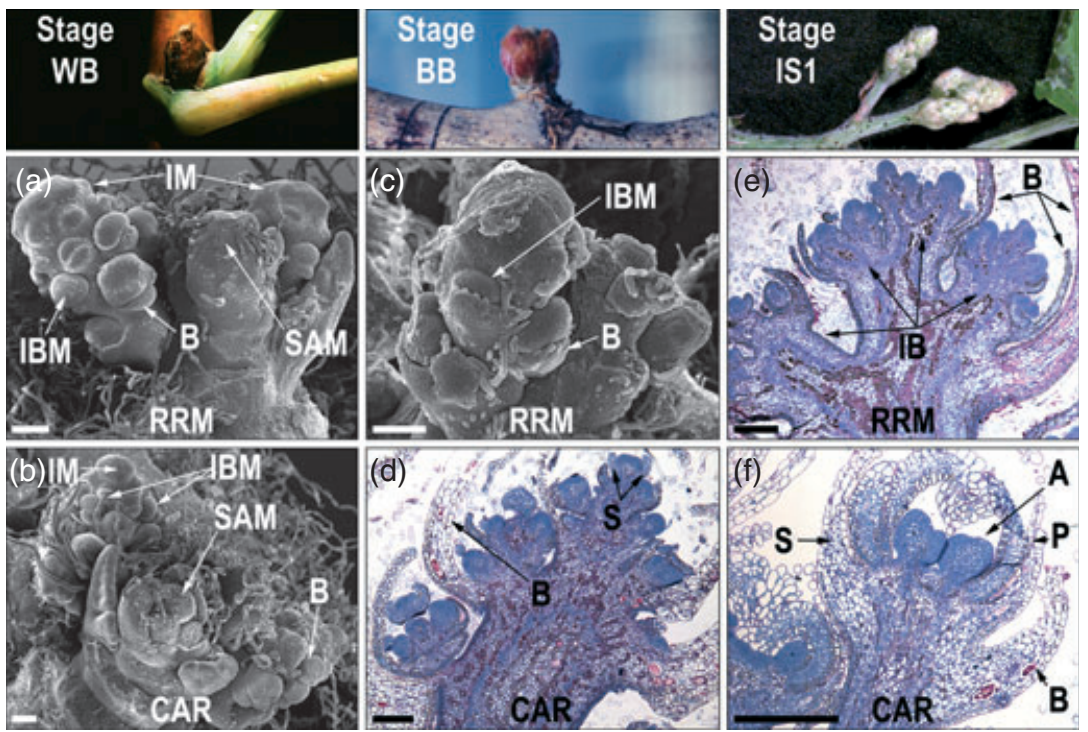

In addition, many RRM flowers displayed alterations of the third and fourth whorls, showing partial transformation of stamens into petaloid structures (Figure S1). Finally, some flowers developed additional internal flowers, probably as a result of the lack of determination of flower meristems (Figure 1e). These abnormal RRM flowers resulted in an unusual opening of the flower from the top of the calyptra (Figures 1e and S1), in contrast to the wildtype flower opening, where the calyptra detaches from the receptacle (Figure 1f).

The early development of RRM and Carignan inflorescences was analysed through optical and scanning electron microscopy, from the initiation of the inflorescence meristem during latent bud organogenesis at year $n-1$ to flower meristem initiation at year $n$. As the RRM plants present a delay in inflorescence differentiation, equivalent bud phenological stages were considered for comparison. During early latent bud formation, RRM plants displayed a similar pattern and timing of inflorescence initiation as Carignan plants (data not shown). However, by the end of inflorescence formation within latent buds, the RRM inflorescences (Figure 2a) did not exhibit the typical conical structure formed by inflorescence branch meristems in Carignan plants (Figure 2b). Later, at bud burst, the RRM inflorescence structure did not show a significant evolution (Figure 2c), whereas Carignan inflorescences already showed the initiation of flower meristems (Figure 2d). At stage IS1, a few RRM inflorescences were just beginning to initiate flower meristems. However, most inflorescence branch meristems were still generating additional inflorescence branches, preventing the differentiation of terminal flowers (Figure 2e). At the same stage, Carignan inflorescences had already formed flowers displaying three well-differentiated whorls: sepals, petals that had begun to fuse, and developing anthers with filaments not yet visible (Figure 2f). Thus, the RRM inflorescences show a delay in the initiation of flower meristems and floral organ differentiation, which is related to the reiterative production of additional inflorescence meristems.

Cluster structure. To characterize the differences in cluster structure between RRM and Carignan plants, we performed a quantitative analysis of their architecture. A schematic diagram of representative clusters for each genotype is shown in Figure 3. Carignan clusters only branched to generate axes of order 2 and 3 ( $A 2$ and $A 3$ ), whereas RRM inflorescences displayed higher branching, producing axes of
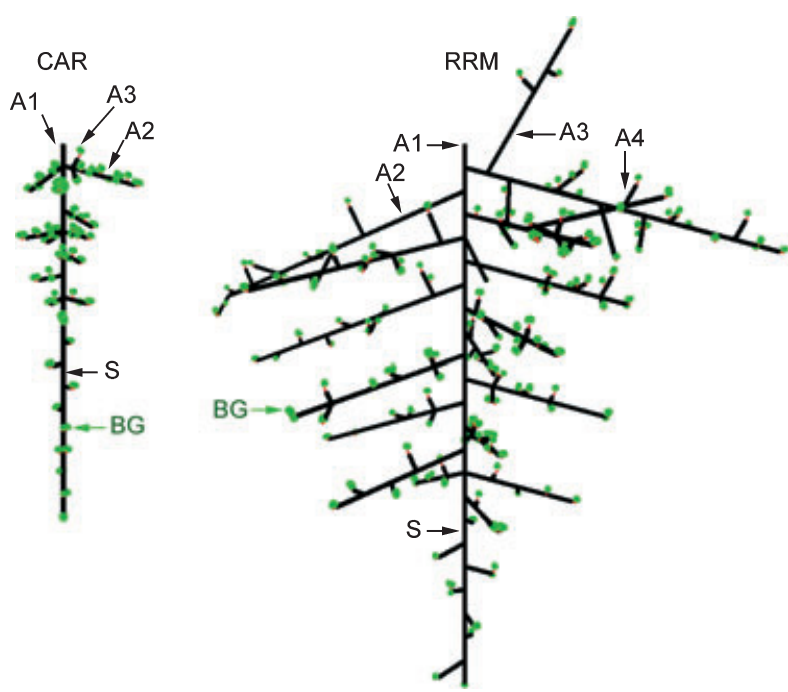

Figure 3. Schematic diagram representing the architecture of Carignan (CAR and reiterated reproductive meristem (RRM) clusters.

$A$, axis; A1, main axis; A2-A4, axes from orders 2 to 4; BG, berry groups; $\mathrm{S}$, segment. Berry groups are represented in green. Lengths of segments have been computed from the measured length of axes assuming that all the segments have the same length. 


\begin{tabular}{llllc}
\hline Trait & Definition & CAR & RRM & $P$ value $^{\text {a }}$ \\
\hline Axis type & \% of A2 & $76.69(16.39)$ & $17.92(4.75)$ & $*$ \\
& $\%$ of A3 & $23.31(16.39)$ & $63.91(10.95)$ & $*$ \\
& $\%$ of A4 & - & $17.54(14.31)$ & - \\
Branching & \% of A5 & - & $0.63(1.4)$ & - \\
Axis length & Cumulative axis length & $17.17(7.63)$ & $126.4(46.55)$ & $*$ \\
& Axis length/A1 length & $26.18(7.19)$ & $152.8(47.52)$ & $*$ \\
Axis density & Axis number/cumulative axis length & $2.47(0.48)$ & $11.87(4.88)$ & $* *$ \\
Branching rate & Axis number/A1 length & $1.66(0.24)$ & $0.82(0.1)$ & ns \\
& & & $9.81(4.48)$ & $* *$ \\
\hline
\end{tabular}

Table 1 Analysis of cluster architecture

Mean value (SD) obtained from the analysis of six Carignan (CAR) and five reiterated reproductive meristems (RRM) clusters: $A 1$, main axis of the cluster; $A 2$, order-2 axes; $A 3$, order-3 axes; $A 4$, order- 4 axes, $A 5$, order- 5 axes; -, no data.

a Statistical significance of mean differences between CAR and RRM according to a Welch's $t$-test:

*highly significant $(P \leq 0.01)$; ${ }^{* *}$ significant $(0.01<P \leq 0.05)$; ns, non-significant $(P>0.05)$.

up to order 5 (A5). As a whole, RRM clusters exhibited a number of axes, and a cumulative axis length that was on average seven and five times longer than in Carignan clusters, respectively (Table 1). The cumulative axes length related to the main cluster axis length remained significantly higher in RRM clusters than in Carignan (Table 1). However, axes density (axis number/cumulative axis length) in the mutant and in the wild type were found to be similar (Table 1), in agreement with the higher branching rate (axis number/A1 length) of RRM clusters (Table 1). Consequently, at ripening stage RRM bunches appeared much larger and less compacted than Carignan ones (Figure S1).

Tendril phenotype. The Vitaceae tendrils have a common ontogenetic origin with inflorescences, and could be considered as sterile reproductive structures adapted to climbing (Boss et al., 2003). RRM plants also displayed a differential phenotype on tendrils, which were more branched than in Carignan plants (Figure $4 a, b$ ). Carignan tendrils generally have two $(56 \%)$ or three $(44 \%)$ branches, whereas in RRM plants, $86 \%$ of tendrils develop three or more branches. Furthermore, RRM plants frequently exhibited leaf/ tendril or shoot/tendril substitutions that are rare in Carignan plants (Figures S1 and 4c). In addition, intermediate leaf/inflorescence structures were also observed in RRM plants (Figure 4d).

\section{Transcriptional analysis of the RRM phenotype}

Considering that the RRM phenotype probably appeared as a consequence of a single mutation in a given cell line, a plausible hypothesis was that the mutation had a dominant effect on the phenotype. Based on this hypothesis we reasoned that the analyses of transcriptional changes associated with phenotype expression could provide information on the gene(s) altered in the somatic variant. We performed a transcriptional analysis of Carignan and RRM inflorescences at two different times of development, on 18 April 2006 and 27 April 2006. Given the delayed development of RRM inflorescences with respect to Carignan ones, at the first date both inflorescences were at stage IS1, whereas at
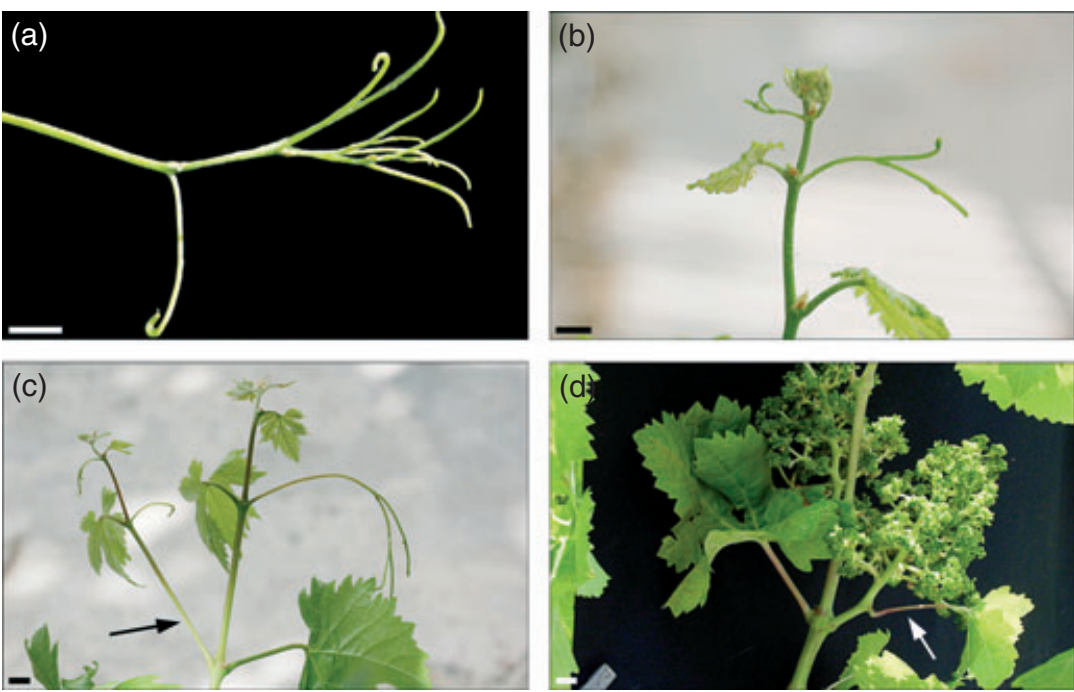

Figure 4. Abnormal development of determined lateral organs in the reiterated reproductive meristem (RRM) somatic variant.

(a) RRM tendril with multiple branches.

(b) Typical Carignan shoot structure, with two branched tendrils opposite to leaves.

(c) Conversion of a tendril into a lateral shoot (arrow) in the RRM genetic background.

(d) Intermediate lateral structure containing one inflorescence and a leaf (arrow) in the RRM genetic background.

Scale bars: $1 \mathrm{~cm}$. 
the second date RRM inflorescences were at stage IS2, and Carignan ones were at stage IS3.

Using the commercially available Grape Affymetrix GeneChip $^{\circledR}$ (Santa Clara, CA), containing 16436 grapevine probe sets, only 58 probe sets were found to be differentially expressed between Carignan (CAR) IS1 and RRM IS1, and/or between CAR IS3 and RRM IS2 (Figure 5a; Table S2). The eight genes exhibiting the highest under-representation in RRM inflorescences encoded transcriptional factors belonging to the YABBY protein family (abnormal floral organs, $A F O)$, as well as several homologs of MADS-box genes SEPALLATA 1 (SEP1), SEP3, APETALA 3 (AP3), PISTILLATA (a)
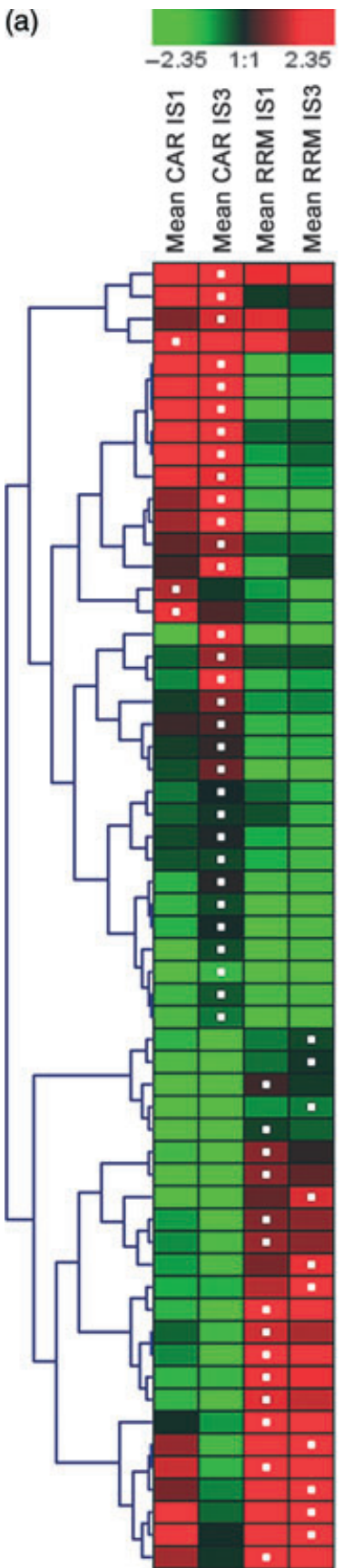

PR protein-CD802429 cytochrome P450-CD713793 phosphatase-CB339435 Thi biosynthetic enzyme-CF 405441 SEP3.BQ792730 PI.CF 373459 AP3.CF414182 AFO-CF372908 SEP1-AF373601 AGLE/13-AF373602 AG/SHP.AF265562 AGIAGL11-CB989581 PR protein-CB970020 phytochrome P450-CF 403395 glycoprotein EP1-CF372454 NHF-BQ800624

hydrolase protein-CB971492 phosphorylase protein-CB970077 strictosidine synthase-CB972058 NHF-CF413969 RD22-CB344488 omega-6 FAD -СB920839 fatty acid elongase-CBO0 4761 oxidoreductase-CD716155 NHF-CF208215

unknown protein-CB972189 $\mathrm{N}$-methyltransferase-CF208335 Protease inhibitor -AY156047 unknown protein-CF 372224 beta-glucosidase-CDO04218 blue copper-like protein-CF415350 NHF-BQ800502

cytochrome P450-CK136955 Lhca5 protein-CF215949 unknown protein-CF516786 phospholipase-CF209511 XTR -CD801116 unknown protein-CF207450 XTR-CF206328 XTR-BQ793580 Betv I protein-CF51252\% TFL1-BQ106731 carbonic anhydrase- $/ 514663$ glucose acyltransfejase -CF511425 XTR-CB980277

TF-CB80277

beta-Amyrin Synthase-CF512552

unknown protein-CF212147

EXL3.CF513065

NHF-CF513139

PRP4-CF568798

unknown protein-CF213268

AGP.CF214588

FLA.CF513613

ABC transporter-CD801577

WTL1-AF003007

WTL1-CB920849

SVP -BQ794993 (b)

\section{VvPI expression}

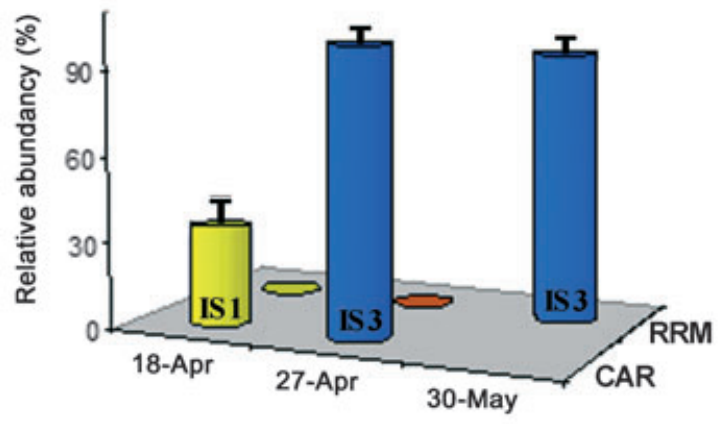

(c)

VvTFL1A expression

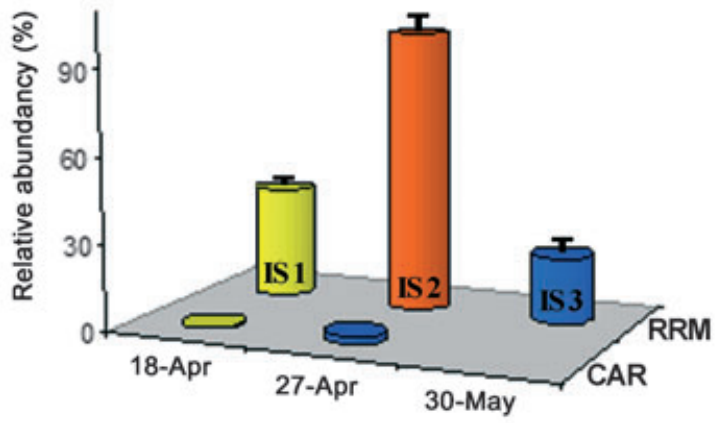

(d)

VvSVP1 expression

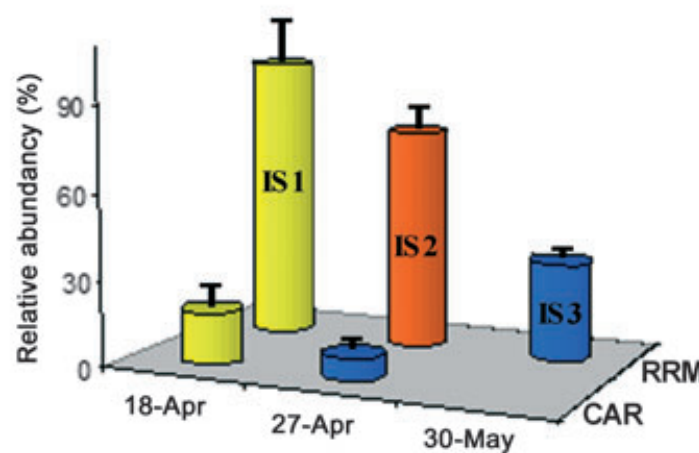

Figure 5. Transcriptional analyses of reiterated reproductive meristem (RRM) and Carignan (CAR) inflorescences.

(a) Hierarchical clustering of genes differentially expressed in CAR and RRM inflorescence developmental stages. Average intensity values are represented by a color scale for samples corresponding to inflorescence stages 1 (IS1), 2 (IS2) and 3 (IS3). White points indicate the sample with absolute maximum expression for a gene (b) VvPI, (c) VvTFL1A and (d) VvSVP1 qRT-PCR expression patterns in CAR and RRM inflorescences at IS1, IS2 and IS3. Expression values were normalized with EF1alpha and expressed as relative abundance (\%). Error bars: SDs for technical triplicates. 
(PI), AGAMOUS (AG)/SHATERPROOF (SHP), AG/AGL11 and $A G L 6 / A G L 13$. Their underexpression in the IS1 and IS2 stages of RRM inflorescence could be a consequence of the lack of flower organ differentiation in these inflorescence stages of the somatic variant, as most of them have been shown to be involved in the specification of flower organ identity. Interestingly, two genes encoding proteins involved in the regulation of flowering induction, phase transition and inflorescence development were overexpressed in the RRM inflorescences: $V V S V P 1$, a homolog of Arabidopsis SHORT VEGETATIVE PHASE MADS-box gene, and $V_{V} T F L 1 A$, homologous with Arabidopsis TERMINAL FLOWER 1.

The results of the transcriptional profiling experiment were validated for two RRM upregulated (VVSVP1 and $\left.V_{V} T F L 1 A\right)$ and one downregulated ( $\left.V v P l\right)$ genes using qRTPCR on the same samples, plus one additional RNA sample corresponding to stage IS3 of RRM inflorescences (harvested on 30 May 2006). RRM inflorescences exhibited no detectable expression of $V v P I$ during stages IS1 and IS2 (Figure 5b). However, at stage IS3, RRM inflorescences showed similar levels of $V v P I$ expression as CAR ones. The qRT-PCR experiment also confirmed the high overexpression of $V_{V} T F L 1 A$ at all stages of RRM inflorescence development, whereas $V_{V T F L} 1 A$ was hardly detectable in CAR inflorescences (Figure 5c). Finally, VvSVP1 was overexpressed in RRM plants at all inflorescence stages analyzed (Figure $5 \mathrm{~d}$ ). The biological roles assigned to these genes in Arabidopsis were related to a delay in flowering transition $(S V P)$, as well as phase transition establishment and inflorescence architecture (TFL1), which made them candidates to be responsible for the RRM phenotype.

\section{Genetic analysis of the RRM phenotype}

To test the hypothesis on the putative role of VVTFL1A or $V_{V} S V P 1$ in the origin of the RRM phenotype, we analyzed the segregation of this phenotype as well as that of the gene allelic variants in selfed progenies derived from RRM and CAR plants. Grapevine plants grown from seeds can take between 3 and 5 years to produce inflorescences and fruits. For this reason, plants were phenotyped on the basis of tendril morphology (see Experimental procedures). The RRM phenotype could be observed in the selfed progeny of RRM plants, indicating that the causal mutation was present in the L2 cell layer, which gives rise to the gametes. Only 23 out of 34 segregating plants could be phenotyped, and their phenotypic segregation fitted a 3:1 Mendelian segregation model, as expected for the presence of a single dominant mutation in a heterozygous state in the RRM somatic variant $\left(\chi^{2}=1.75, P=0.18\right)$ (Table 2). Dominance of the RRM phenotype was in agreement with the hypothesis that it could result from the overexpression of VVTFL1A, VVSVP1 or other genes detected in the transcriptional profiling.
Table 2 Segregation of reiterated reproductive meristem (RRM) phenotype and VVTFL1A and VvSVP1 genotypes in the selfed progeny of the RRM somatic variant

\begin{tabular}{lllll}
\hline Genotype & N & WT $^{*}$ & RRM $^{*}$ & ND $^{*}$ \\
\hline SVP1a/SVP1a & 8 & 1 & 5 & 2 \\
SVP1a/SVP1b & 17 & 1 & 8 & 8 \\
SVP1b/SVP1b & 9 & 1 & 7 & 1 \\
Total & 34 & 3 & 20 & 11 \\
TFL1Aa/TFL1Aa & 15 & & 13 & 2 \\
TFL1Aa/TFL1Ab & 14 & & 7 & 7 \\
TFL1Ab/TFL1Ab & 5 & 3 & & 2 \\
Total & 34 & 3 & 20 & 11 \\
\hline
\end{tabular}

*Phenotypes were scored as wild-type (WT), RRM or not determined (ND), following the procedure described in Experimental procedures.

Contrary to plant model systems such as Arabidopsis or tomato (Solanum lycopersicum), grapevine plants are highly heterozygous at most of their loci. Therefore, we analyzed the co-segregation of the RRM phenotype with the two alleles (named as a and b) of VVTFL1A and VVSVP1 present in CAR (Table 2). When considering VVSVP1, which in grapevine is located on linkage group 7 (IASMA, http://genomics. research.iasma.it/iasma/), the three expected genotypes segregated following a 1:2:1 model $\left(\chi^{2}=0.058, P=0.97\right)$ as expected for co-dominant markers. No VVSVP1 allele was found to co-segregate with the RRM phenotype. Regarding the VVTFL1A gene, located on linkage group 6 (R. Velasco, personal communications), the observed genotype segregation was distorted, and did not fit the 1:2:1 model $\left(\chi^{2}=6.94, P=0.03\right)$. However, there was a complete association of the RRM phenotype with the presence of the VVTFL1Aa allele in either the homozygous or heterozygous state, whereas all plants with a wild-type tendril phenotype were homozygous for the VvTFL1Ab allele. In fact, among the 11 individuals that showed the most extreme leafy tendril phenotype, eight were homozygous for VvTFL1Aa. As plants homozygous for the $V_{v} T F L 1 A a$ allele in the segregating progeny from CAR did not display the RRM phenotype, we hypothesized that the VVTFL1Aa allele should have mutated in the L2 cell layer of the CAR plant that gave rise to the somatic variant displaying the RRM dominant phenotype.

\section{The RRM phenotype is caused by a class-II transposon insertion}

Transposon characterization. In order to identify the molecular change responsible for the RRM phenotype, we sequenced 4308 bp for each VvTFL $1 A$ allele, including 3104 and $1204 \mathrm{bp}$ before and after the ATG sequence, respectively. The two CAR alleles showed $98 \%$ of sequence identity with the corresponding Pinot Noir genome sequence (Jaillon et al., 2007). The VVTFL1A coding region included four exons of 201, 63, 41 and $217 \mathrm{bp}$, separated by three introns of 83,467 and $107 \mathrm{bp}$, as previously described 


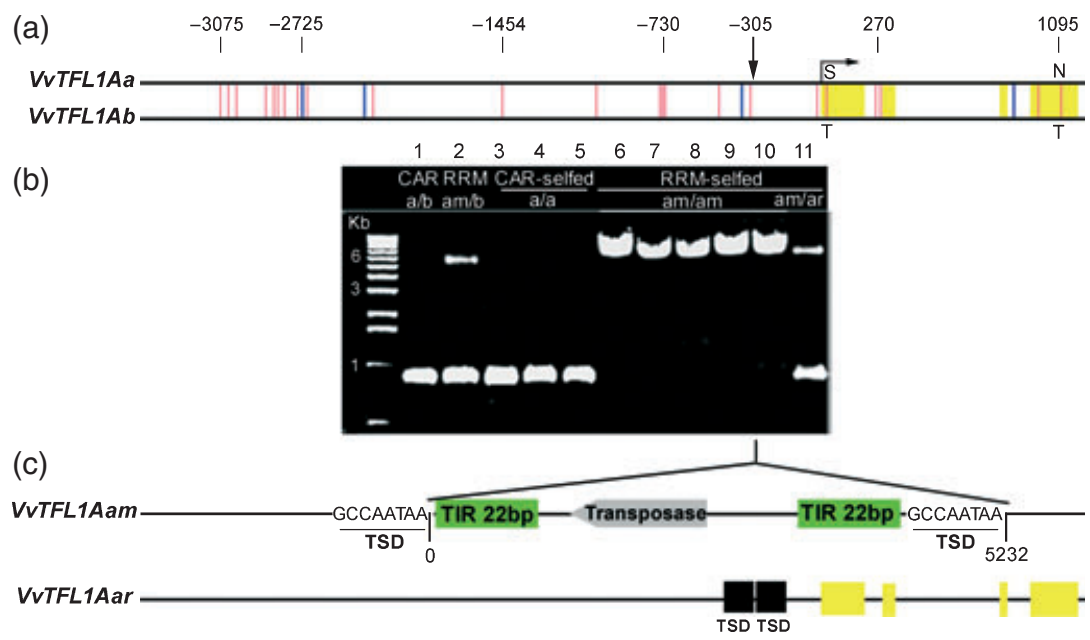

Figure 6. Sequence analysis of the VVTFL1A locus.

(a) Genomic organization of the VVTFL1A locus in Carignan. Exons are represented by yellow boxes. Polymorphism between $V_{V} T F L 1 A a$ and $V v T F L 1 b$ alleles are indicated by pink and blue lines for nucleotide substitutions and INDELs, respectively. Amino acid changes between both alleles are also indicated. The vertical arrow indicates the position of the transposon insertion in the reiterated reproductive meristem (RRM) VvTFLAam allele. The horizontal arrow marks the position of the ATG start codon.

(b) Genotype of $V v T F L 1 A$ alleles in Carignan (CAR), RRM and selfed plants using TFL1-F4/R4 primers. The allele $V v T F L 1 A$ combination is indicated in the second line. Lane 11 shows the bands amplified in a plant carrying a revertant (VvTFL 1Aar) allele. A $5-\mu$ l portion of each PCR product was loaded onto a $0.7 \%$ agarose gel. Line 1 contains the 1-kb DNA ladder from Invitrogen (http://www.invitrogen.com).

(c) Organization of the VVTFL1Aam and VVTFL1Aar mutant alleles found in the RRM-derived plants. The VVTFL1Aam allele bears a 5.2-kb transposon insertion at position $-305 \mathrm{bp}$, with respect to the ATG start codon. Target site duplications (TSDs), terminal inverted repeats (TIRs) and the transposase coding sequence are schematized. VvTFL1Aar has lost the transposon insertion, and presents an 8-bp insertion corresponding to the TSD or transposon footprint.

(Carmona et al., 2007). Twenty seven polymorphisms differentiated the two VVTFL1A allele sequences present in CAR, including four INDELs. Among them, three SNPs were found in the coding region, one in exon 1 and two in exon 4 , giving rise to two amino acid substitutions (Figure 6a).

As expected, the VVTFL1Ab allele sequences were identical in homozygous plants derived from the RRM somatic variant, or from CAR selfing. Regarding $V V T F L 1 A a$, its sequence in homozygous plants derived from RRM selfing was also identical to that obtained in CAR-derived plants, with the exception of a region upstream of the ATG, where we detected the presence of a new approximately 5-kb insertion not present in the CAR VVTFL1Aa allele. This allele was detected in all the individuals showing the RRM phenotype, and was named VVTFL1Aam. All plants genotyped as homozygous for the VVTFL1Aam mutant allele in the RRM-selfed progeny showed the unique presence of this insertion (Figure 6b). However, we detected one plant genotyped as homozygous for VvTFL1Aam in which a PCR amplicon corresponding in size to the non-mutant allele was also amplified. This unexpected amplicon was sequenced and found to correspond to a new allele, VvTFL1Aar, that had lost the inserted DNA, and contained a short tandem duplication of $8 \mathrm{bp}$ (Figure $6 \mathrm{~b}, \mathrm{c}$ ). Thus, some cells of the leaves used to extract DNA in this plant had lost the transposon insertion in one of the two copies of the gene.

The complete sequencing of the inserted DNA revealed a 5232-bp sequence, flanked by an 8-bp target site duplication
(TSD) and 22-bp terminal inverted repeats (TIRs) (CA AGGATTGAAATATCGGTAAA), typical of class-II transposable elements (Figure 6c). BLASTX sequence comparisons with sequence databases showed that the sequenced fragment has a high sequence similarity with Arabidopsis $h A T$ transposons (NP188371). This element was named Hatvine1-rrm, and showed the highest identity (99.9\%) with a Pinot Noir sequence localized on chromosome 5 (scaffold 64, position 1431 877-1 437 144; Genoscope, http:// www.cns.fr). The closest homolog to the predicted protein encoded by Hatvine1-rrm is the Vitis protein CAN71355, which contains the hAT family dimerization domain conserved in transposase-like proteins (Marchler-Bauer et al., 2007). However, no expressed sequence tag (EST) corresponding to this transposase could be found in grapevine databases. Therefore, the RRM phenotype, associated with VVTFL1A overexpression, seems to be related to the insertion of a Hatvine1 element in the promoter region of VvTFL1A. Although we could not confirm that this element is an autonomous element encoding a functional transposase, it mobilizes in the genome of CAR, and has generated at least two new VVTFL1A alleles (VVTFL1Aam and VvTFL1Aar).

Molecular effect of Hatvine1-rrm insertion. Transposon insertions frequently cause loss-of-function alleles. However, the dominant nature of the RRM phenotype suggested the presence of a gain-of-function mutation, in agreement 


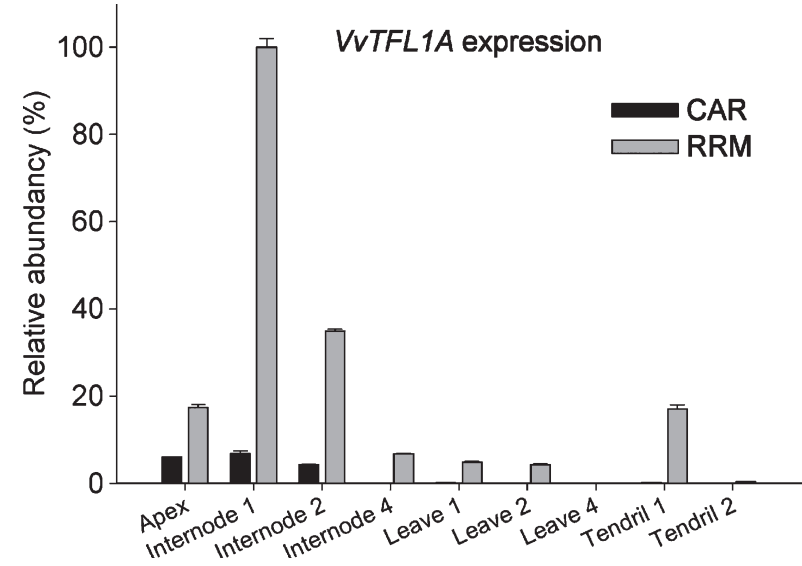

Figure 7. $V_{V} T F L 1 A$ expression along the shoot. $V v T F L 1 A$ qRT-PCR expression analysis along Carignan (CAR) and reiterated reproductive meristem (RRM) shoots. Numbers correspond to the node position from the apex. Expression values were normalized with EF1alpha and expressed as relative abundance (\%).

Error bars: SDs for technical triplicates.

with $V v T F L 1 A$ overexpression in RRM inflorescences. To understand the effect of the Hatvine1-rrm insertion, we analyzed its consequences on VVTFL1A expression. We first determined the organ-specific expression of $V v T F L 1 A$ in the apical part of the shoot using qRT-PCR. VVTFL 1A expression was detected in the CAR apex and the first shoot internodes, whereas it was undetectable in developing leaves and tendrils of the shoot apical region (Figure 7). By contrast, RRM shoots exhibited much higher VVTFL1A expression than CAR in apex and internodes, but also in first developing leaves and tendrils. Both in CAR and RRM plants VvTFL1A expression was reduced with distance from the apex. Thus, the transposon insertion seemed to be related to an enhancement of $V V T F L 1 A$ expression in the shoot apical organs, but its expression remained under developmental control.

We hypothesized that if the transposable element was the cause for the observed overexpression of $V_{V} T F L 1 A$ in the apical parts of the shoot, this expression should be allelespecific, and restricted to the allele carrying the insertion in its promoter region. To test this hypothesis, we developed allele-specific qRT PCR assays and analyzed the expression of both $V_{V} T F L 1 A$ alleles in the first shoot internode of heterozygous plants. As shown in Figure 8, the VvTFL1Aa allele was overexpressed 10-fold in RRM and derived selfed plants when compared with CAR (Figure 8b), whereas no significant differences were detected between expression levels of the VVTFL1Ab allele (Figure 8c). This result supports the hypothesis that the transposon insertion is related to VVTFL $1 A$ overexpression.

To analyze the transcriptional initiation site (TIS) of each allele, we performed $5^{\prime}$ rapid amplification of cDNA ends (RACE) of VvTFL1Aam, VvTFL1Aa and VvTFL $1 b$ transcripts in plants homozygous for each allele, derived from selfing of either RRM or CAR genotypes. These experiments identified the same TIS position for the three different
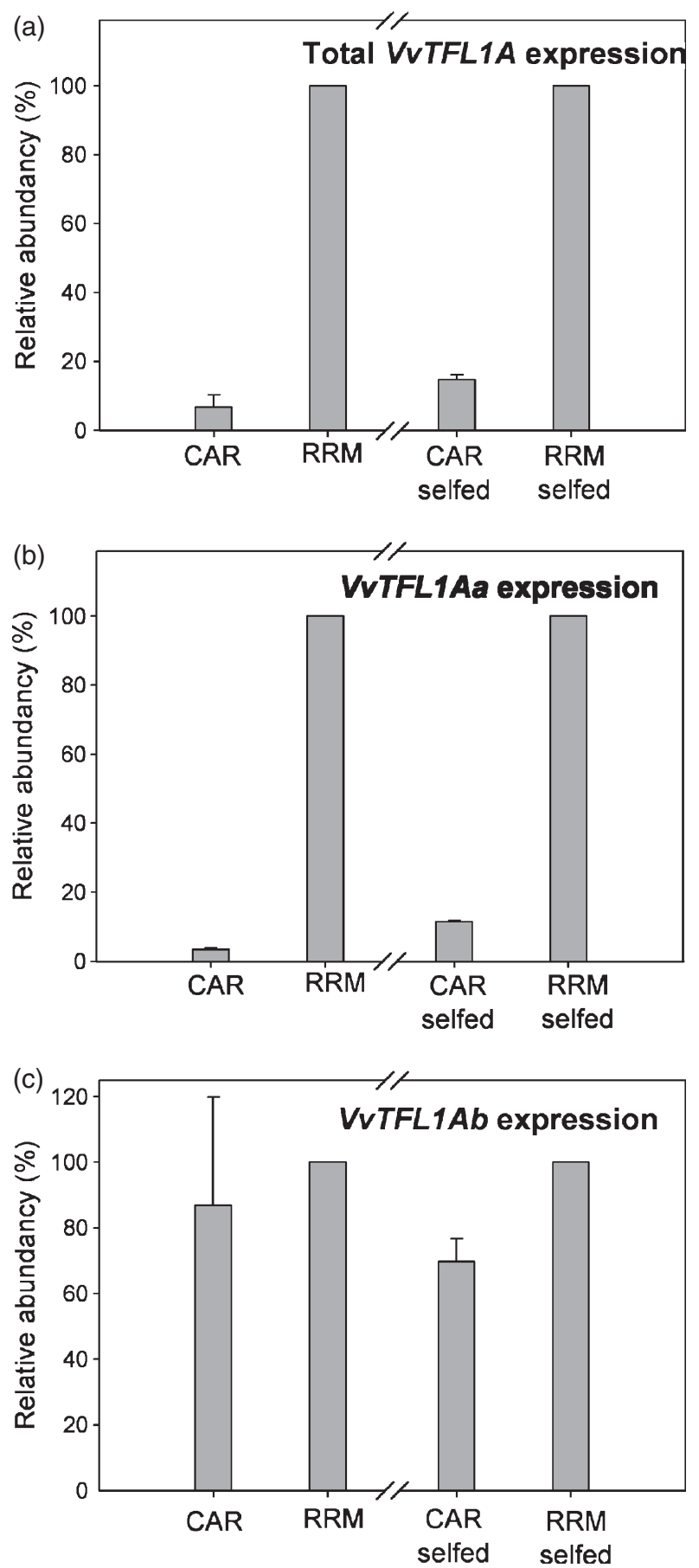

Figure 8. $V V T F L 1 A$ allelic expression.

(a) Total VVTFL1A, (b) VVTFL1Aa and (c) VVTFL1Ab qRT-PCR expression in internode stem tissue of Carignan (CAR), reiterated reproductive meristem (RRM), and of CAR- and RRM-selfed segregants heterozygous for the VVTFL1A locus. Expression values were normalized with EF1alpha, and were expressed as relative abundancies (\%). To facilitate comparison, expression in RRM and RRM-selfed plants were arbitrarily fixed to $100 \%$.

Error bars: SDs for biological duplicates. 
alleles $(a, b$ and $a m), 83 \mathrm{bp}$ upstream of the ATG, indicating that VVTFL1Aam-specific overexpression did not result from the use of a new TIS related to the Hatvine1-rrm insertion. Therefore, these results suggest that the overexpression of the VVTFL1Aam allele is caused by an enhancer effect resulting from the close insertion of Hatvine1-rrm in its promoter region.

\section{DISCUSSION}

\section{The RRM phenotype is associated with the enhancement of VvTFL1A expression caused by a class-II transposon insertion}

Here, we report on the identification of the molecular basis of the RRM phenotype, first detected as a spontaneous sport in grapevine plants of cultivar CAR (Chatelet et al., 2007), which has a large effect on inflorescence and fruit cluster development. Genetic analyses in selfed progenies of the original somatic variant showed that the phenotype was transmitted to the progeny as a single dominant trait. Furthermore, a comparative morphological and transcriptional analysis performed in CAR and RRM plants permitted the identification of two overexpressed genes ( $V V T F L 1 A$ and $V_{v}$ SVP1) as candidates to be responsible for the RRM phenotype. Co-segregation of a specific VVTFL1Aa allele with the RRM phenotype, and the identification of the Hatvine1rrm transposon inserted in position -305 bp of its promoter region in $\mathrm{RRM}$ plants suggested that this insertion was the origin of the phenotypic alteration. This hypothesis is strongly supported by the fact that overexpression of VVTFL1A in the somatic variant is allele-specific, and only affects the VVTFL1Aam allele that carries the transposon insertion in cis. Still, we cannot completely exclude the unlikely possibility that another linked mutation with a cis effect on VVTFL1A expression could be the origin of the RRM phenotype.

Hatvine1-rrm belongs to the family of $h A T$ transposable elements recently characterized in grapevine (Benjak et al., 2008). The $h A T$ superfamily is widely distributed in eukaryotes (Rubin et al., 2001) and is the most prevalent transposon class in the grapevine genome, with over 1500 related sequences (Benjak et al., 2008). The element identified in the VVTFL1Aam promoter belongs to the subfamily Hatvine1, and its presence demonstrates the existence of active transposition for members of this subfamily in the grapevine genome. Transposition activity is supported by the detection of both new insertion events, i.e. the one described in the VvTFL1Aam allele, and excision events, which leave behind the 8-bp duplication footprint, as shown by the generation of the VVTFL1Aar allele. The transposase open reading frame (ORF) in this Hatvine1 element seems to be potentially complete, and able to encode a putative functional protein. However, Hatvine 1-rrm could also be mobilized by a master copy acting in trans. Insertion of the Hatvine1-rrm element caused a dominant effect associated with the overexpression of the VVTFL1Aam tagged allele. Overexpression of this allele was not constitutive, but took place in a developmental-specific manner, being observed in young developing organs along the stem, including the shoot apex, leaves, internodes and tendrils, and progressively decreasing in older organs. As the insertion orientation placed the Hatvine1 transposase ORF opposite to the VVTFL1A ORF, both genes should be expressed through independent transcriptional units. Furthermore, our experimental results show that the transcription start of the VvTFL1Aam allele in RRM is the same as that of the VVTFL1Aa allele in CAR. All these data support the conclusion that Hatvine1-rrm enhances the expression of the VVTFL1Aam allele, either by enhancing transcription initiation at its own transcription start site, or alternatively by disrupting a repressor binding site on the VVTFL1Aam promoter. However, no information is available on the existence of such sites for the promoter of TFL1 homologs in other species. The wide literature on class-II transposable elements in plants reports frequent cases of insertion in $5^{\prime}$ upstream gene regulatory sequences, which generally negatively affect gene expression (Yang et al., 2005; Xu et al., 2007; Zhai et al., 2008), and there are only a few examples in which transposon-mediated sequence rearrangements have generated new gene expression patterns (Lister et al., 1993; Kloeckener-Gruissem and Freeling, 1995). Positive regulation or enhancement of gene expression mediated by transposable elements located not necessarily in $5^{\prime}$ upstream regions has been observed (Bradley et al., 1993; Greene et al., 1994) as a result of cis-enhancing effects, depending on a trans-acting active transposon (Greene et al., 1994), or probably as a result of sequences carried over by transposable elements that could act as positive regulatory motifs (Dooner and Weil, 2007). Mechanisms of cis-activation tagging promoted by endogenous transposable elements, like the one described for Hatvine1rrm, could generate gain-of-function mutations, and consequently new somatic phenotypes in perennial species. These phenotypes could be directly exposed to natural selection, providing genetic variation for adaptation along the vegetative cycle of perennial species, which could also be transmitted to the next sexual and/or asexual generations. Further analyses will be required to test these possibilities.

\section{Role of VvTFL1A in grapevine development}

The RRM phenotype is associated with the enhanced expression of grapevine VVTFL1A in the apical shoot organs. VVTFL1A is the closest grapevine homolog to Arabidopsis TERMINAL FLOWER 1 (TFL1) (Bradley et al., 1997), Antirrhinum CENTRORADIALIS (CEN) (Bradley et al., 1996), pea (Pisum sativum) DETERMINATE (DET) (Singer et al., 1990), tomato SELF-PRUNING (SP) (Pnueli et al., 1998), tobacco (Nicotiana tabacum) CET (Amaya et al., 1999) or Lolium 
perenne LpTFL1 (Jensen et al., 2001), encoding a protein similar to animal phosphatidylethanolamine-binding proteins (PEBPs), the molecular function of which is not completely understood (Keller et al., 2004; Odabaei et al., 2004), although it seems to be associated with trafficking to protein storage vacuoles (Sohn et al., 2007). The analyses of mutant phenotypes in Arabidopsis, Antirrhinum and in other species support a biological function of this gene in the control of the length of plant developmental phases, as well as in the maintenance of indeterminate growth of inflorescence meristems (Schultz and Haughn, 1991; Bowman et al., 1993). Consequently, loss-of-function mutants commonly show significantly shorter vegetative phases than wild-type plants, as well as rapid differentiation of terminal flowers in place of inflorescence meristems (Shannon and MeeksWagner, 1991; Alvarez et al., 1992; Schultz and Haughn, 1993; Bradley et al., 1997). In addition, overexpression of these genes in transgenic plants generates highly branched inflorescence phenotypes, resulting from a delay in the differentiation of flowers, as well as a delay in developmental phase transitions (Ratcliffe et al., 1998).

Three $V_{V} T F L 1$-related genes have been identified in grapevine under the names VVTFL1A, VVTFL1B and VVTFL1C (Carmona et al., 2007). Interestingly, overexpression of VVTFL $1 A$ in transgenic Arabidopsis plants had similar effects as those described for the ectopic expression of TFL1 (Ratcliffe et al., 1998), in terms of increasing inflorescence branching and delaying the initiation of flower meristems (Boss et al., 2006; Carmona et al., 2007). Futhermore, the RRM phenotype linked to VVTFL1A overexpression in grapevine is also coincident with the phenotype described in Arabidopsis. The effects of enhanced expression of VVTFL1A could be mediated in part by VVSVP1, also overexpressed in the RRM somatic variant. This MADS-box gene was identified in Arabidopsis as encoding a dosagedependent repressor of flowering (Hartmann et al., 2000; Lee et al., 2007; Liu et al., 2007), and directly represses transcription of the floral integrator SOC1 gene in the shoot apex (Li et al., 2008). Constitutive overexpression of TFL 1 in transgenic Arabidopsis causes a delay in flowering time as a result of an extension of the vegetative phase (Ratcliffe et al., 1998). However, it is not clear whether TFL 1 produces this effect through the positive regulation of SVP expression in Arabidopsis.

Altogether, the available information indicates that initiation of lateral shoot structures in grapevine requires a certain level of VVTFL1A, as suggested by previous expression analyses (Joly et al., 2004; Boss et al., 2006; Carmona et al., 2007), but also indicates that VVTFL1A should be progressively downregulated to complete flower and tendril differentiation. This hypothesis on the role of $V V T F L 1 A$ in grapevine would predict that loss-of-function mutations or transgenic silenced plants would generate minimal lateral organs, such as tendrils or inflorescences, with no ramifica- tion, and a rapid differentiation of flowers, possibly in a single dichasium structure. Further experiments will be required to confirm this prediction.

Grapevine fruit cluster size and shape is initially determined by the structure of inflorescence and its elongation during cluster growth (Shavrukov et al., 2003). Furthermore, the number of flowers formed per inflorescence meristem, together with the success of pollination and fruit set, determine the number of fruits that, depending on their size, will also affect the cluster compactness. Very little is known about the genetic control of cluster size and shape. As shown by the results of the cluster structural analysis in RRM and CAR plants, changes in VVTFL1A expression of the level observed in the RRM somatic variant could affect axis length and branching pattern of the cluster, and consequently its size and shape, by increasing or decreasing the order number of branch axes, and their total number. TFL 1 has been proposed to play a key role in the evolution of inflorescence architecture (Prusinkiewicz et al., 2007), and VVTFL1A could play a relevant role in the control of this trait in grapevine, and consequently on cluster structure. The large differences existing in terms of cluster size and shape between wild ( $V$. vinifera ssp. sylvestris) and cultivated ( $V$. vinifera ssp. sativa) grapevine genotypes suggest that cluster traits have been selected during the domestication of grapevine (This et al., 2006). Further experiments will be required to determine the participation of VVTFL1A in the control of genetic variation for cluster size and shape in grapevine, and to test whether specific VVTFL1A variants have been selected during the domestication process.

\section{EXPERIMENTAL PROCEDURES}

\section{Plant materials}

The RRM somatic variant appeared as a sport of the grapevine cultivar Carignan ( $V$. vinifera L.), also known by its synonyms Cariñena and Mazuelo. Their genetic identity was previously confirmed (Chatelet et al., 2007), and both the somatic variant and the original CAR plant are conserved in the grapevine collection at the INRA Domaine de Vassal (http://www1.montpellier.inra.fr/vassal/). Both genotypes were propagated in containers as woody canes taken from the original plants. Herbaceous buds (HBs) were sampled in April between the 15th and 21st nodes from the basis of the shoot, winter buds (WBs) were collected in December between the fourth and 10th nodes from the basis of the shoot, and buds at budburst (BBs) corresponded to the buds where first leaf tissue was visible. Inflorescence developmental stages were identified as described in Sreekantan et al. (2006). They corresponded to very young inflorescences (no single flower visible) emerging from the bud (IS1), clearly separated from the vegetative axis (IS2) and showing the first single flower (IS3). Vegetative organs were collected from shoots with more than six nodes separating the apex, tendrils or tendril-like organs, leaves and internodes.

To study the genetic control of the RRM phenotype we generated two segregating populations by selfing the wild-type CAR plant (CAR-selfed progeny), as well as the RRM somatic variant (RRMselfed progeny), further established in pots in the glasshouse. Plants were phenotyped for alterations observed on tendrils. Plants 
with the presence of leafy tendril structures and tendrils with more than three branches were classified as the RRM type because of the lack of those phenotypes in the selfed CAR segregating plants. Only three plants in the control segregating population produced three-branched tendrils at low frequency. In this context, plants that developed more than $50 \%$ of three-branched tendrils per shoot were classified as the RRM phenotype in the RRM segregating progeny. To avoid erratic phenotyping, plants with less than $50 \%$ of three-branched tendrils were not classified (not determined), and were kept for further analysis. Finally, plants that only developed two-branched tendrils were classified as the wild type. Phenotype was based on the observation of a minimum of six tendrils per shoot, and was repeated in at least two shoots for 12 and 11 individuals from CAR- and RRM-selfed progenies. The goodness-of-fit to expected segregation ratios was assessed by a chi-square test.

\section{Microscopy analysis}

For scanning electron microscopy (SEM) and histological analyses, samples were processed as described by (Chatelet et al., 2007).

\section{Analysis of cluster architecture}

To study cluster architecture, a sample of six wild-type and five mutant clusters were selected at ripening stage. The multiscale coding method defined by Godin et al. (1997) was applied to model cluster architecture into a multiscale tree graph (MTG). Three organization levels were considered: (1) rachis (R), (2) axes (A), and (3) segments (S) and berry groups (BG). Axis length was scored and integrated as attributes of $A$. Based on these MTGs, several architectural traits were extracted using AMAPMOD (Godin and Guédon, 2003). Topological differences between the two genotypes were illustrated by schematic 3D representations of the clusters using the GEOM module of AMAPMOD (Godin and Guédon, 2003), and are represented in two dimensions in Figure 3.

\section{Gene expression analyses}

Grapevine RNA was extracted following the protocol of Reid et al. (2006), and further purified with Qiagen RNeasy Mini columns (http://www.qiagen.com), including a DNase treatment, as recommended by the manufacturer.

Microarrays. Transcriptional profiling was performed on early inflorescence developmental stages for the CAR and the RRM plants. Three biological replicates were processed per experiment. Synthesis of labeled probes, hybridization and scanning of the GeneChip ${ }^{\circledR}$ Vitis vinifera (Grape) Genome array v1.0 (Affymetrix ${ }^{\circledR}$, http://www.affymetrix.com) were carried out at the Genomics Service of the CNB (http://www.cnb.uam.es/ genomica/). Microarray data were first normalized using the Affymetrix normalization package available on GEPAS (http://gepas.bioinfo.cipf.es/). Normalization included Robust MultiArray Average (RMA) for background correction (Irizarry et al., 2003), perfect match (PM) correction (Li and Wong, 2001), quantile array standardization (Bolstad et al., 2003) and finally median polish procedure (Tukey, 1977). A Student's $t$-test was performed on RMA expression values from inflorescence samples harvested at the same date in RRM and CAR: CAR IS1 versus RRM IS1, and CAR IS3 versus RRM IS2. A multiple-test correction was applied to the $P$ value of the $t$-statistics to adjust the false discovery rate (Benjamini and Hochberg, 1995). Genes with an adjusted $P<0.05$ and an expression change fold ratio of 1.5 or more between RRM and CAR were selected for further analysis. The normalized data set was clustered via hierarchical clustering using the average linkage method and the
Pearson uncentered distance metric using Genesis 1.7.2 (Sturn et al., 2002).

$q R T-P C R$. Analyses were performed as described by Fernandez et al. (2007) using an ABI PRISM 7300 Sequence Detection System (Applied Biosystems, http://www.appliedbiosystems.com) with SYBR $^{\circledR}$ Green to detect dsDNA synthesis. Specific primers were designed in the $3^{\prime}$ untranslated region of VVTFL1A (AF378127) and VvSVP1 (B0794993) (Table S1: TFL1F, TFL1R, SVPF and SVPR). Amplification of $\mathrm{VvPI}$ (D0059750) and VvEF1alpha (BQ799343) genes was performed using previously described primers (Fernandez et al., 2007).

Allele-specific expression for VVTFL1A was analyzed by SYBR Green-based qRT-PCR during 30 cycles of PCR amplification. Forward-specific primers for allelic discrimination assays were designed as recommended by Gupta et al. (2005). Primer sequences are available in Table S1 (TFL1R, 1095FA and 1095FC). Genomic DNA and cDNA from homozygous segregant individuals were used to verify the allelic discrimination efficiency in control experiments.

$5^{\prime}$ RACE. For the $5^{\prime}$ RACE, we used $1 \mu \mathrm{g}$ of total RNA of both VVTFL1Aa and VVTFL1Ab homozygous individuals of the CAR- and RRM-selfed progenies using the SMART RACE cDNA Amplification kit (Clontech, http://www.clontech.com), as recommended by the manufacturer. TFL1R primer (Table S1) was used as gene-specific primer 1 (GSP1). 5'-RACE products were purified and sequenced with TFL1R primer, as described below.

\section{Molecular genetics analyses}

Genomic DNA was extracted from young leaves using the Qiagen Dneasy Plant Mini kit (Qiagen), as recommended by the manufacturer.

Genotyping. Cleaved amplified polymorphic site (CAPS) markers were used to detect SNP 1095 of VVTFL1A using Apol, and SNP 3332 of $V v S V P 1$ using Taql. Primers are listed in Table S1 $\left(\right.$ TFL1F $^{1095}$, TFL1R, SVP1F ${ }^{3332}$ and SVP1R ${ }^{3332}$ ).

Sequencing. To sequence the different alleles of VVTFL1A, six sets of specific primers were designed to amplify 700-900-bp overlapping sequences in the promoter and ORF of $V_{v} T F L 1 A$ (chromosome un-random, scaffold 87, position $137062691-$ 137067 029; Genoscope, http://www.genoscope.cns.fr/spip/Vitisvinifera-e.html) (Table S1: TFL1-F1-F6 and TFL1-R1-R6). PCR amplifications were performed from 10 to $20 \mathrm{ng}$ of grape genomic DNA using the AmpliTaq DNA polymerase (Applied Biosystems), as recommended by the manufacturer. The transposon amplicon was amplified using TFL1-F4 and TFL1-R4 primers. PCR amplifications were performed from 15 to $30 \mathrm{ng}$ of grape genomic DNA using the LongRange PCR Enzyme mix (Qiagen), as recommended by the manufacturer. PCR products were treated with Exosap-IT reagent, as recommended by the manufacturer, and were sequenced at the Genomic Service of the Parque Cientifico de Madrid, in an ABI prism 3730 DNA sequencer (Applied Biosystems).

The transposon was first sequenced with TFL1-F4 and TFL1-R4, giving the $5^{\prime}$ and $3^{\prime}$ end transposon sequence. The identification of closely related sequences was made against the Genoscope Blat server, to find a homolog reference sequence. Thereafter, 20 primers were defined along the reference sequence and used to sequence the Hatvine 1-rrm transposon product. In total, 17 primers were used to sequence $3 X$ transposon coverage (Table S1: TE-F1F10 and TE-R1-R7). Base calling, quality trimming and alignment of 
$A B I$ chromatograms was performed using SEOSCAPE 2.5. Sequence polymorphisms were verified manually.

\section{ACKNOWLEDGEMENTS}

The authors want to thank Diego Lijavetzky for help with Affymetrix chips analysis, Philippe Chatelet for helping with inflorescence fixations, Gilbert Lopez and the CNB glasshouse facility staff for the care of the plants, and the CNB Genomics service staff for Affymetrix GeneChip hybridization experiments. Franscisco Madueño and Desmond Bradley are acknowledged for their critical reading of the manuscript, and we thank Carlos Alonso Blanco and María José Carmona for helpful discussions. This research was supported by grants BIO2005-07612 and BIO2008-03892 from the Spanish Ministry for Science and Innovation, and by funds from the INRA Plant Breeding and Genetics Department.

\section{SUPPORTING INFORMATION}

Additional Supporting Information may be found in the online version of this article:

Figure S1. Additional phenotypic features of the reiterated reproductive meristem (RRM) somatic variant.

Table S1. Primer sequences used in this work.

Table S2. Genes identified by transcriptional analysis that are differentially expressed in inflorescences of Carignan (CAR) and the RRM somatic variant.

Please note: As a service to our authors and readers, this journal provides supporting information supplied by the authors. Such materials are peer-reviewed and may be re-organized for online delivery, but are not copy-edited or typeset. Technical support issues arising from supporting information (other than missing files) should be addressed to the authors.

\section{REFERENCES}

Alvarez, J., Guli, C.L., Yu, X.H. and Smyth, D.R. (1992) Terminal flower: a gene affecting inflorescence development in Arabidopsis thaliana. Plant J. 2 , 103-116.

Amaya, I., Ratcliffe, O.J. and Bradley, D.J. (1999) Expression of CENTRORADIALIS (CEN) and CEN-like genes in tobacco reveals a conserved mechanism controlling phase change in diverse species. Plant Cell, 11, 1405-1418.

Benjak, A., Forneck, A. and Casacuberta, J.M. (2008) Genome-wide analysis of the "cut-and-paste" transposons of grapevine. PLoS ONE 3, e3107.

Benjamini, Y. and Hochberg, Y. (1995) Controlling the false discovery rate: a practical and powerful approach to multiple testing. J. Roy. Soc. Statist. Soc. Ser. B 57, 289-300.

Bolstad, B.M., Irizarry, R.A., Astrand, M. and Speed, T.P. (2003) A comparison of normalization methods for high density oligonucleotide array data based on variance and bias. Bioinformatics, 19, 185-193.

Boss, P.K. and Thomas, M.R. (2002) Association of dwarfism and flora induction with a grape 'green revolution' mutation. Nature, 416, 847-850.

Boss, P.K., Buckeridge, E.J., Poole, A. and Thomas, M.R. (2003) New insights into grapevine flowering. Funct. Plant Biol. 30, 593-606.

Boss, P.K., Sreekantan, L. and Thomas, M.R. (2006) A grapevine TFL homologue can delay flowering and alter floral development when overexpressed in heterologous species. Funct. Plant Biol. 33, 31-41.

Bowman, J.L., Alvarez, J., Weigel, D., Meyerowitz, E.M. and Smyth, D.R. (1993) Control of flower development in Arabidopsis thaliana by APETALA1 and interacting genes. Development, 119, 721-743.

Bradley, D., Carpenter, R., Sommer, H., Hartley, N. and Coen, E. (1993) Complementary floral homeotic phenotypes result from opposite orientations of a transposon at the plena locus of Antirrhinum. Cell, 72, 85-95.

Bradley, D., Carpenter, R., Copsey, L., Vincent, C., Rothstein, S. and Coen, E. (1996) Control of inflorescence architecture in Antirrhinum. Nature, 379, 791-797.

Bradley, D., Ratcliffe, O., Vincent, C., Carpenter, R. and Coen, E. (1997) Inflorescence commitment and architecture in Arabidopsis. Science, 275, 80-83.
Carmona, M.J., Calonje, M. and Martinez-Zapater, J.M. (2007) The FT/TFL1 gene family in grapevine. Plant Mol. Biol. 63, 637-650.

Chatelet, P., Laucou, V., Fernandez, L., Sreekantan, L., Lacombe, T., Martinez Zapater, J.M., Thomas, M.R. and Torregrosa, L. (2007) Characterization of Vitis vinifera L. somatic variants exhibiting abnormal flower development patterns. J. Exp. Bot. 58, 4107-4118.

Dooner, H.K. and Weil, C.F. (2007) Give-and-take: interactions between DNA transposons and their host plant genomes. Curr. Opin. Genet. Dev. 17, 486492

Fernandez, L., Romieu, C., Moing, A., Bouquet, A., Maucourt, M., Thomas M.R. and Torregrosa, L. (2006) The grapevine fleshless berry mutation. A unique genotype to investigate differences between fleshy and nonfleshy fruit. Plant Physiol. 140, 537-547.

Fernandez, L., Torregrosa, L., Terrier, N., Sreekantan, L., Grimplet, J., Davies, C., Thomas, M.R., Romieu, C. and Ageorges, A. (2007) Identification of genes associated with flesh morphogenesis during grapevine fruit development. Plant Mol. Biol. 63, 307-323.

Godin, C. and Guédon, Y. (2003) AMAPmod: Exploring and Modeling Plant Architecture. Version 2.1.16. Montpellier: CIRAD/INRA UMR Modélisation des plantes.

Godin, C., Guédon, Y., Costes, E. and Caraglio, Y. (1997) Measuring and analyzing plants with the AMAPmod software. In 2nd International Sym posium on Computer Challenges in Life Sci (Michalewicz, M., ed.). Melbourne: CSIRO, pp. 53-84.

Greene, B., Walko, R. and Hake, S. (1994) Mutator insertions in an intron of the maize knotted1 gene result in dominant suppressible mutations. Genetics, 138, 1275-1285.

Gupta, M., Yates, C.R. and Meibohm, B. (2005) SYBR Green-based real-time PCR allelic discrimination assay for beta2-adrenergic receptor polymorphisms. Anal. Biochem. 344, 292-294.

Hartmann, U., Hohmann, S., Nettesheim, K., Wisman, E., Saedler, H. and Huijser, P. (2000) Molecular cloning of SVP: a negative regulator of the floral transition in Arabidopsis. Plant J. 21, 351-360.

Irizarry, R.A., Hobbs, B., Collin, F., Beazer-Barclay, Y.D., Antonellis, K.J., Scherf, U. and Speed, T.P. (2003) Exploration, normalization, and summaries of high density oligonucleotide array probe level data. Biostatistics, 4, 249-264.

Jaillon, O., Aury, J.M., Noel, B. et al. (2007) The grapevine genome sequence suggests ancestral hexaploidization in major angiosperm phyla. Nature 449, 463-467.

Jensen, C.S., Salchert, K. and Nielsen, K.K. (2001) A TERMINAL FLOWER1-like gene from perennial ryegrass involved in floral transition and axillary meristem identity. Plant Physiol. 125, 1517-1528.

Joly, D., Perrin, M., Gertz, C., Kronenberger, J., Demangeat, G. and Masson, J.E. (2004) Expression analysis of flowering genes from seedling-stage to vineyard life of grapevine cv. Riesling. Plant Sci. 166, 1427-1436.

Keller, E.T., Fu, Z. and Brennan, M. (2004) The role of Raf kinase inhibitor protein (RKIP) in health and disease. Biochem. Pharmacol. 68, 10491053.

Kloeckener-Gruissem, B. and Freeling, M. (1995) Transposon-induced promoter scrambling: a mechanism for the evolution of new alleles. Proc. Natl Acad. Sci. USA, 92, 1836-1840.

Kobayashi, S., Goto-Yamamoto, N. and Hirochika, H. (2004) Retrotransposoninduced mutations in grape skin color. Science, 304, 982.

Lee, J.H., Yoo, S.J., Park, S.H., Hwang, I., Lee, J.S. and Ahn, J.H. (2007) Role of SVP in the control of flowering time by ambient temperature in Arabidopsis. Genes Dev. 21, 397-402.

Li, C. and Wong, W.H. (2001) Model-based analysis of oligonucleotide arrays: expression index computation and outlier detection. Proc. Natl Acad. Sci. USA, 98, 31-36.

Li, D., Liu, C., Shen, L., Wu, Y., Chen, H., Robertson, M., Helliwell, C.A., Ito, T., Meyerowitz, E. and Yu, H. (2008) A repressor complex governs the integration of flowering signals in Arabidopsis. Dev. Cell, 15, 110-120.

Lijavetzky, D., Ruiz-Garcia, L., Cabezas, J.A., De Andres, M.T., Bravo, G. Ibanez, A., Carreno, J., Cabello, F., Ibanez, J. and Martinez-Zapater, J.M. (2006) Molecular genetics of berry colour variation in table grape. Mol. Genet. Genomics, 276, 427-435.

Lister, C., Jackson, D. and Martin, C. (1993) Transposon-induced inversion in Antirrhinum modifies nivea gene expression to give a novel flower color pattern under the control of cycloidearadialis. Plant Cell, 5, 15411553. 
Liu, C., Zhou, J., Bracha-Drori, K., Yalovsky, S., Ito, T. and Yu, H. (2007) Specification of Arabidopsis floral meristem identity by repression of flowering time genes. Development, 134, 1901-1910.

Marchler-Bauer, A., Anderson, J.B., Derbyshire, M.K. et al. (2007) CDD: a conserved domain database for interactive domain family analysis. Nucleic Acids Res. 35, D237-D240.

McGovern, P.E., Voigt, M.M., Glusker, D.L. and Exner, L.J. (1986) Neolithic resinated wine. Nature, 381, 480-481.

Moore, G.A. (2001) Oranges and lemons: clues to the taxonomy of Citrus from molecular markers. Trends Genet. 17, 536-540.

Odabaei, G., Chatterjee, D., Jazirehi, A.R., Goodglick, L., Yeung, K. and Bonavida, B. (2004) Raf-1 kinase inhibitor protein: structure, function, regulation of cell signaling, and pivotal role in apoptosis. Adv. Cancer Res. 91, 169-200.

Pnueli, L., Carmel-Goren, L., Hareven, D., Gutfinger, T., Alvarez, J., Ganal, M., Zamir, D. and Lifschitz, E. (1998) The SELF-PRUNING gene of tomato regulates vegetative to reproductive switching of sympodial meristems and is the ortholog of CEN and TFL1. Development, 125, 1979-1989.

Prusinkiewicz, P., Erasmus, Y., Lane, B., Harder, L.D. and Coen, E. (2007) Evolution and development of inflorescence architectures. Science, 316, $1452-1456$.

Ratcliffe, O.J., Amaya, I., Vincent, C.A., Rothstein, S., Carpenter, R., Coen, E.S. and Bradley, D.J. (1998) A common mechanism controls the life cycle and architecture of plants. Development, 125, 1609-1615

Reid, K.E., Olsson, N., Schlosser, J., Peng, F. and Lund, S.T. (2006) An optimized grapevine RNA isolation procedure and statistical determination of reference genes for real-time RT-PCR during berry development. BMC Plant Biol. 6, 27.

Rubin, E., Lithwick, G. and Levy, A.A. (2001) Structure and evolution of the hAT transposon superfamily. Genetics, 158, 949-957.

Schultz, E.A. and Haughn, G.W. (1991) LEAFY, a homeotic gene that regulates inflorescence development in Arabidopsis. Plant Cell, 3, 771-781.

Schultz, E.A. and Haughn, G.W. (1993) Genetic analysis of the floral initiation process (FLIP) in Arabidopsis. Development, 119, 745-765.

Shannon, S. and Meeks-Wagner, D.R. (1991) A mutation in the Arabidopsis TFL1 gene affects inflorescence meristem development. Plant Cell, 3, 877892.
Shavrukov, Y.N., Dry, I.B. and R., T.M. (2003) Inflorescence and bunch architecture development in Vitis vinifera L. Aust. J. Grape Wine Res. 10, 116124.

Singer, S.R., Hsuing, L.P. and Huber, S.C. (1990) Determinate (det) mutant of Pisum sativum L. (Leguminosae: Papilionoideae) exhibits an indeterminate growth pattern. Am. J. Bot. 77, 1330-1335.

Sohn, E.J., Rojas-Pierce, M., Pan, S., Carter, C., Serrano-Mislata, A., Madueño, F., Rojo, E., Surpin, M. and Raikhel, N.V. (2007) The shoot meristem identity gene TFL 1 is involved in flower development and trafficking to the protein storage vacuole. Proc. Natl Acad. Sci. USA, 104, 18801-18806.

Sreekantan, L., Torregrosa, L., Fernandez, L. and Thomas, M.R. (2006) VVMADS9, a class B MADS-box gene involved in grapevine flowering, shows different expression patterns in mutants with abnormal petal and stamen structures. Funct. Plant Biol. 33, 877-886.

Sturn, A., Quackenbush, J. and Trajanoski, Z. (2002) Genesis: cluster analysis of microarray data. Bioinformatics, 18, 207-208.

This, P., Lacombe, T. and Thomas, M.R. (2006) Historical origins and genetic diversity of wine grapes. Trends Genet. 22, 511-519.

Tukey, J.W. (1977) Exploratory Data Analysis. Massachusetts: AddisonWesley Publishing.

Walbot, V. and Evans, M.M. (2003) Unique features of the plant life cycle and their consequences. Nat. Rev. Genet. 4, 369-379.

Xu, L., Wang, L., Liu, T., Qian, W., Gao, Y. and An, C. (2007) Triton, a novel family of miniature inverted-repeat transposable elements (MITEs) in Trichosanthes kirilowii Maximowicz and its effect on gene regulation. Biochem. Biophys. Res. Commun. 364, 668-674.

Yang, G., Lee, Y.H., Jiang, Y., Shi, X., Kertbundit, S. and Hall, T.C. (2005) A two-edged role for the transposable element Kiddo in the rice ubiquitin2 promoter. Plant Cell, 17, 1559-1568.

Zhai, J., Liu, J., Liu, B., Li, P., Meyers, B.C., Chen, X. and Cao, X. (2008) Small RNA-directed epigenetic natural variation in Arabidopsis thaliana. PLoS Genet. 4, e1000056.

Zohary, D. (2004) Unconscious selection and the evolution of domesticated plants. Econ. Bot. 58, 5-10.

Zohary, D. and Hopf, M. (2000) Domestication of Plants in the Old World. Oxford: Oxford University Press.

Accession Numbers: the Hatvine1-rrm sequence has been deposited in the NCBI database under accession number FJ538308; Carignan VVTFL1Aa and VVTFL1Ab sequences have been deposited in the NCBI database under accession numbers GQ368243 and GQ368244, respectively; and Affymetrix expression profiling can be found in ArrayExpress under accession number E-MEXP-1950. 